\title{
A 31 Year Old Lady with Post-Partum Systemic Lupus Erythematosus and Lupus Nephritis
}

\author{
Malaweera $\mathrm{A}^{1 *}$ and Huang $\mathrm{LL}^{2}$ \\ ${ }^{1}$ Department of Medicine, Monash University, Australia \\ 2Department of Nephrology, Box Hill Hospital, Australia
}

*Corresponding author: Malaweera A, Department of Medicine, Eastern Health Clinical School and Monash University, Box Hill Hospital, 8 Arnold Street, Box Hill, 3123, Victoria, Australia, Tel: +61413104081; Email: arunimalaweera@gmail.com / aruni.malaweera@easternhealth.org.au

\section{Abstract}

Introduction: Systemic lupus erythematosus (SLE) is an autoimmune systemic disease that presents heterogeneously with multiple organ involvement with varying severity. It is more common in women of child bearing age and majority of studies have noted increased SLE activity during pregnancy, causing peri-partum complications like pre-eclampsia, preterm labour and stillbirth. Our case describes a young female patient presenting post-partum with florid signs and symptoms of diffuse SLE. This highlights the importance of considering autoimmune disease as part of a wide differential diagnosis; to aid prompt diagnosis with thorough clinical assessment, laboratory autoimmune panel testing and management of the organ disease with an appropriate level of immunosuppression, to improve the overall disease prognosis.

Case Presentation: We present a 31-year-old woman who suffered pre-eclampsia during her pregnancy, presenting one month postpartum with haemolytic anaemia, decompensated cardiomyopathy with serositis and acute renal impairment. She was diagnosed with systemic lupus erythematosus with lupus nephritis and started on immunosuppressive therapy with hydroxychloroquine, corticosteroids and mycophenolate mofetil. After treatment, she has had improvement in her renal impairment, haemolytic anaemia, serositis and her autoimmune markers.

Conclusion: This case highlights the highly heterogeneous presentation of SLE and its close interaction between pregnancy and its outcomes. It is important to have a low threshold for consideration of autoimmune diseases like SLE in a young female presenting with above symptoms. The overall management of an individual with SLE should be carried out in a multidisciplinary team involving physicians of different specialties, nursing and allied health staff and psychologists. 
Abbreviations: SLE: Systemic Lupus Erythematosus; ANA: Anti-Nuclear Antibody; dsDNA: Double Stranded DNA Antibody; ITP: Immune Thrombocytopenic Purpura; ECG: Electrocardiogram; CXR: Chest Xray; USS: Ultrasound of Chest; MMF: Mycophenolate Mofetil.

\section{Introduction}

Systemic lupus erythematosus (SLE) is a chronic autoimmune disease affecting multiple organs. Its predominance in women, especially of child bearing age is characteristic for the disease. It is extremely heterogeneous with a wide range of clinical and serological manifestations and marked disease course in different individuals. Although SLE can present commonly with constitutional symptoms like fatigue, weight loss and arthralgia, $25-50 \%$ patients can have serious organ involvement with lupus nephritis, pleural disease and myopericarditis. Additionally, SLE activity is associated with poor pregnancy and peri-partum outcomes with high risk of pre-eclampsia, pregnancy loss, pre-term birth, stillbirth and low birth weight [1]. SLE is characterised by anaemia, hypocomplementemia and raised anti-nuclear antibody (ANA) and double stranded DNA antibody (dsDNA), the latter being diagnostic. Today we present a challenging case of a 31-year-old presenting one month post-partum with anaemia, cardiomyopathy with a pericardial effusion, pleural effusions, arthralgia and acute renal failure.

\section{Case Report}

We present a 31-year-old Caucasian female (G1P1) who delivered twins through normal vaginal delivery 6 weeks prior to presenting to hospital with SLE. At 34 weeks, she was diagnosed as having pre-eclampsia with evidence of proteinuria, hypertension and deranged liver function tests. As a result, she underwent chemical induction and delivered twins via normal vaginal delivery without complications. She was hypertensive at $>140 / 80$ $\mathrm{mmHg}$ in the peri-partum period and was discharged with labetalol.

Her past medical history included immune thrombocytopenic purpura (ITP) with previous steroid use and was currently in remission. She was in a family of four, with a history of SLE in both her brother and mother. Two weeks after childbirth, she developed fatigue, shortness of breath on exertion and bilateral leg swelling and was diagnosed as having pleural effusions on chest x-ray. She received frusemide for this and her labetalol was changed to nifedipine. She was also found to have iron deficiency and anaemia with haemoglobin of $68 \mathrm{~g} / \mathrm{L}$ and was given an iron infusion. Other than mild vaginal bleeding post-delivery, she denied any other sources of bleeding. Two weeks following this, she developed arthralgia in the small joints of the hands and a macular, blanching rash over her feet, knees, chest wall and hands but sparing the face [2].

She presented to a public hospital for further assessment and management of her anaemia and fluid overload. On clinical assessment, she had evidence of conjunctival pallor and a macular blanching rash over chest, hands and feet. She also had small joint synovitis with swelling and tenderness at proximal interphalangeal joints and pitting odema of the feet. Her vital observations were stable throughout the admission with blood pressures of $120-130 \mathrm{mmHg}$ systolic and $70-90 \mathrm{mmHg}$ diastolic.

She had a normocytic anaemia of $\mathrm{Hb} 71 \mathrm{~g} / \mathrm{L}$, with a blood film consisting of mild anisocytosis with moderate normochromic normocytic anaemia with occasional tear drop and rare fragmented red cells. Her haemolysis screen revealed a positive coombs test, a low haptoglobin and high reticulocytes consistent with a haemolytic anaemia. Cross-matching studies also found that multiple positive antibodies making it difficult to find compatible blood for transfusion.

She also had acute kidney injury with a creatinine of $160 \mu \mathrm{mol} / \mathrm{L}$, hyperkalaemia with potassium of $6 \mathrm{mmol} / \mathrm{L}$, metabolic acidosis with bicarbonate of $16 \mathrm{mmol} / \mathrm{L}$ and a 24-hour urinary protein excretion of $0.64 \mathrm{~g}$ (Table 1 ). Her kidney ultrasound revealed normal size kidneys without obstruction. Her urinalysis revealed 15 million/L erythrocytes without any cellular casts. Additionally, she also had evidence of serositis. This was in the form of bibasal (left>right) pleural effusions (Figures 1 \& 2) and a moderate pericardial effusion with moderately-severe reduction in LV function on an Echocardiogram (Echo). She had a serum troponin 68ng/L $(<14 \mathrm{ng} / \mathrm{L})$ and a BNP was $3500 \mathrm{pmol} / \mathrm{L} \quad(<15.3 \mathrm{pmol} / \mathrm{L})$ and her Electrocardiogram (ECG) revealed normal sinus rhythm. 


\section{Open Access Journal of Urology \& Nephrology}

\begin{tabular}{|c|c|c|c|c|c|c|c|c|c|c|}
\hline & Day 1 & Day 2 & Day 3 & Day 4 & Day 5 & Day 6 & Day 7 & Day 8 & Day 9 & Day 10 \\
\hline $\mathrm{K} \mathrm{(mmol/L)}$ & 6 & 5.7 & 6.1 & 5.1 & 4.7 & 4.1 & 4.2 & 5 & 4.5 & 3.8 \\
\hline Urea (mmol/L) & 18 & 20 & 22.5 & 28.4 & 25.6 & 21.3 & 15.1 & 14.1 & 14.3 & 14.4 \\
\hline Creatinine (mmol/L) & 160 & 179 & 185 & 168 & 139 & 137 & 95 & 113 & 102 & 93 \\
\hline GFR (mL/min/1.73m2) & 36 & 32 & 30 & 34 & 43 & 44 & 68 & 55 & 63 & 70 \\
\hline $\mathrm{Hb}(\mathrm{g} / \mathrm{L})$ & 71 & 72 & 67 & 66 & 57 & 55 & 65 & 94 & 92 & 89 \\
\hline ACR (mg/mmol) & 136.5 & \multicolumn{10}{|l|}{} \\
\hline PCR (g/mmol) & 0.18 &
\end{tabular}

Key $\mathrm{K}=$ potassium, $\mathrm{U}=$ urea, Creat = creatinine, eGFR = estimated glomerular filtration rate $(\mathrm{CKD}-\mathrm{EPI}), \mathrm{Hb}=$ haemoglobin, $\mathrm{ACR}=$ albumin to creatinine ratio, $\mathrm{PCR}=$ protein to creatinine ratio.

Table 1: Laboratory results during hospitalization.
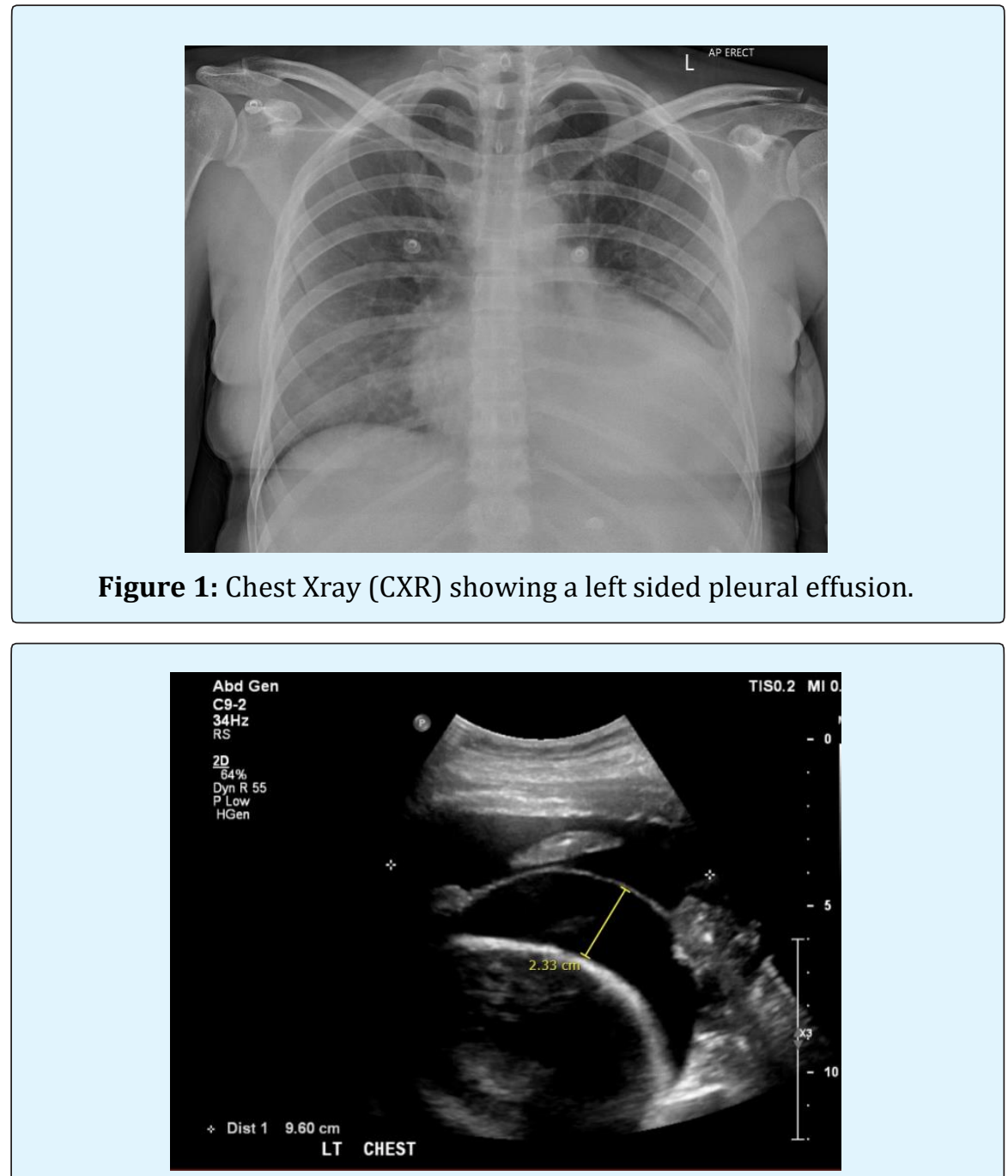

Figure 2: Ultrasound of Chest (USS) showing a left sided pleural effusion. 
Her family history of SLE, pre-eclampsia during pregnancy and her current presentation with arthralgia, serositis, haemolytic anaemia and acute renal impairment, prompted an autoimmune screen (Table 2). Her ANA was homogenously positive with a $>2560$ titre with reduced $\mathrm{C} 3$ and $\mathrm{C} 4$ of $0.33 \mathrm{~g} / \mathrm{L}$ and $0.03 \mathrm{~g} / \mathrm{L}$, respectively. She also had a high dsDNA level of 4485
$\mathrm{IU} / \mathrm{ml}$ confirming the diagnosis of SLE. Due to the presence of severe haemolytic anaemia with multiple antibodies precluding a blood transfusion and shortness of breath making it difficult for the patient to lie supine, a renal biopsy was abandoned and she was empirically started on treatment.

\begin{tabular}{|c|c|c|}
\hline Autoimmune screen & Patient Value & Normal Value \\
\hline ANA & Positive. Homogenous $>2560$ titre & Negative \\
\hline dsDNA (IU/ml) & 4485 & $<100 \mathrm{IU} / \mathrm{ml}$ \\
\hline C3 level (g/L) & 0.33 & $0.9-1.8 \mathrm{~g} / \mathrm{L}$ \\
\hline C4 level (g/L) & 0.03 & $0.1-0.4 \mathrm{~g} / \mathrm{L}$ \\
\hline ANCA (units/ml) & $<5$ & $<5$ units $/ \mathrm{ml}$ \\
\hline RF (kunits/L) & $<10$ & $<10$ kunits $/ \mathrm{L}$ \\
\hline ENA & Negative & Negative \\
\hline Anti PLA2R (RU/ml) & $<2$ & $<2 \mathrm{RU} / \mathrm{ml}$ \\
\hline ESR $(\mathrm{mm} / \mathrm{hr})$ & 117 & $10-15 \mathrm{~mm} / \mathrm{hr}$ \\
\hline CRP (mg/L) & 29 & $<5 \mathrm{mg} / \mathrm{L}$ \\
\hline Lupus anticoagulant & Negative & Negative \\
\hline Reticulocytes \% & $6.50 \%$ & $0.5-2 \%$ \\
\hline Haptoglobin $(\mathrm{g} / \mathrm{L})$ & $<0.10 \mathrm{~g} / \mathrm{L}$ & $0.3-2 \mathrm{~g} / \mathrm{L}$ \\
\hline Bilirubin $(\mu \mathrm{mol} / \mathrm{L})$ & 2 & $22 \mu \mathrm{mol} / \mathrm{L}$ \\
\hline Direct coombs test & Positive & Negative \\
\hline
\end{tabular}

Key $\mathrm{ANA}=$ antinuclear antigen, dsDNA= Double stranded Deoxyribonucleic acid, $\mathrm{C} 3=$ complement 3 level, C4= complement 4 level, ANCA= Anti Neutrophil Cytoplasmic Antibody, RF= Rheumatoid Factor, ENA= Extractable Nuclear Antigen, Anti PLA2R= Anti Phospholipase A2 antibodies, ESR= erythrocyte sedimentation rate, CRP= C-reactive protein.

Table 2: Autoimmune, haemolytic and thrombophilia panel showing evidence of SLE and haemolytic anaemia.

She was given three pulses of intravenous methylprednisolone $500 \mathrm{mg}$ followed by prednisolone 50 $\mathrm{mg}$ and hydroxychloroquine $200 \mathrm{mg}$ daily. She was also started on mycophenolate mofetil (MMF) $1 \mathrm{~g}$ twice daily as immunosuppression for Lupus Nephritis. Two units of packed red cells were given for her anaemia once she was able to be cross-matched, while her cardiomyopathy was managed with Bisoprolol and Frusemide. The rest of her inpatient stay was unremarkable but showed an improvement in her renal function from a peak creatinine of $185 \mu \mathrm{mol} / \mathrm{L}$ to $93 \mu \mathrm{mol} / \mathrm{L}$.

\section{Discussion}

Systemic lupus erythematosus is a multi-system autoimmune disease with a heterogeneous presentation. It occurs up to 10 times more commonly in women, particularly women of child bearing age [3]. It can present with a wide range of symptoms and signs include a rash, arthritis, serositis, nephritis and neuropsychological disturbance with psychosis and seizures. Constitutional symptoms like fatigue and fever seems to be the most common during presentation, rates being $50-90 \%$ [4].
Arthritis and arthralgia are also common, occurring in up to $90 \%$ of patients with a characteristic polyarticular, symmetrical distribution without erosion [5]. Skin manifestations include the classic malar butterfly rash occurring up to $50 \%$, discoid skin lesions, urticaria and photosensitivity.

Concerningly, the morbidity and mortality in SLE is determined by its multi-organ involvement and dysfunction. Renal involvement in the most apparent and can occur in up to $40 \%$ of SLE patients [6]. The presentation and extent of disease is highly variable, ranging from asymptomatic haematuria/proteinuria to rapidly progressive glomerulonephritis. The pathogenesis is said to be due to deposition of immune complexes involving dsDNA antibodies on various sites in the kidney, namely the mesangium and the subendothelial region [7]. Normally, patients present with a raised creatinine, proteinuria and evidence of urinary sediment with cellular casts alongside elevation of dsDNA levels and hypocomplementemia. A kidney biopsy is indicated in most patients with lupus nephritis to establish a diagnosis but patients with $<500 \mathrm{mg} /$ day of proteinuria and bland 
urinary sediment may not require a kidney biopsy. After biopsy, lupus nephritis is divided into categories

\begin{tabular}{|c|c|c|}
\hline Class & Type of abnormality & Management \\
\hline I & Minimal mesangial Lupus Nephritis & Monitoring \\
\hline II & Mesangial proliferate Lupus Nephritis & $\begin{array}{c}\text { Generally, a RAAS inhibitor but can consider } \\
\text { immunosuppressive therapy for proteinuria }>1 \mathrm{~g} / 24 \text { hours }\end{array}$ \\
\hline \multirow{4}{*}{ III } & Focal Lupus Nephritis (involving $<50 \%$ glomeruli) & \multirow{4}{*}{$\begin{array}{l}\text { Induction and Maintenance therapy with steroids and } \\
\text { MMF/Cyclophosphamide }\end{array}$} \\
\hline & III A: active lesions & \\
\hline & III A/C: active and chronic lesions & \\
\hline & III C: chronic lesions & \\
\hline \multirow{4}{*}{ IV } & Diffuse Lupus Nephritis (involving $>50 \%$ glom & \multirow{4}{*}{$\begin{array}{l}\text { Induction and Maintenance therapy with steroids and } \\
\text { MMF/Cyclophosphamide }\end{array}$} \\
\hline & IV A: active lesions & \\
\hline & IV A/C: active and chronic lesions & \\
\hline & IV C: chronic lesions & \\
\hline $\mathrm{V}$ & Membranous Lupus Nephritis & Consider steroids for proteinuria $>3 g / 24$ hours \\
\hline $\mathrm{VI}$ & Advanced sclerosing Lupus Nephritis & Preparation of Renal replacement therapy \\
\hline
\end{tabular}

Table 3: Classes of Lupus Nephritis according to Renal Biopsy.

Pulmonary involvement is also common in SLE, occurring in up to $90 \%$ of patients on autopsy [8]. It manifests as pleurisy and exudative pleural effusions with a high LDH8. Cardiac disease in SLE patients can involve many areas of the heart, notably pericarditis +/- effusion being the most common in up to $25 \%$ of the patients [9]. Other cardiac manifestations include myocarditis and coronary artery disease. Gastrointestinal involvement occurs in up to $40 \%$ patients and can result in oesophagitis, pseudo-obstruction, hepatitis, pancreatitis and mesenteric ischemia [10].

Haematological manifestations are common in SLE and span from anaemia, thrombocytopenia and lymphopenia. The causes of anaemia in SLE patients could be multifactorial with anaemia of chronic disease due to chronic inflammation being the most common. Other causes include iron deficiency, aplastic anaemia and autoimmune haemolytic anaemia. Autoimmune haemolytic anaemia has a prevalence of about 10\% in SLE patients $[11,12]$ and is associated with other significant organ dysfunction including renal, cardiac and pleural disease. This type of anaemia presents with increased reticulocyte count, raised $\mathrm{LDH}$, raised indirect bilirubin, low haptoglobin and a positive coombs test. Interestingly, a phenomenon called Evans syndrome exists where there is occurrence of two or more haematological immune cytopenias, with ITP and AIHA combination being the most common. In addition, in $50 \%$ of these patients, this could be a manifestation of SLE and present before the onset of SLE [11-13]. A study on 26 patients in 2006 looking at treatment of severe autoimmune haemolytic anaemia in SLE patients found that corticosteroids were the best first line of treatment for the anaemia [14].
Neuropsychiatric manifestations can not only be frequent in up to $90 \%$ patients with SLE but can be on a wide spectrum from peripheral neuropathies, psychosis, seizures to cognitive dysfunction [15].

The relationship between and pregnancy and SLE is interesting and has been thoroughly reviewed in literature. The effect of SLE on fertility is debatable. Although some studies have suggested a reduction in fertility amongst SLE patients due to age, less ovarian reserve with cyclophosphamide, disease activity and concurrent antiphospholipid syndrome16-18, others have not observed differences in fertility due to good disease control prior to conception and less use of cyclophosphamide [16-19].

Another concept debated in the literature is SLE activity in pregnancy. Whilst some studies found no significant increase in SLE activity between matched pregnant and non-pregnant SLE patients, majority of the studies found high SLE activity in pregnant patients. These studies collectively demonstrated a 2-3-fold increase in SLE activity during pregnancy, most being mild-moderate disease activity. The pathophysiology of this could be due to $\mathrm{T}$ cell activity. A healthy pregnancy leads to a shift from $\mathrm{TH} 1$ to $\mathrm{TH} 2$ mediated immune response, enabling the mothers' immune tolerance to the foetus. SLE is considered a TH2 mediated disease, therefore may worsen during pregnancy [20]. Risk factors for this seem to be active disease before conception and multiple flares in the years prior to conception2. It is observed that SLE flares can occur anytime during pregnancy and importantly, several months after delivery. 
However, literature on SLE and negative pregnancy outcomes has been consistent. SLE has been associated with an increased risk of pre-eclampsia, pregnancy loss, pre-term labour, stillbirth and low birth weight. Preeclampsia rates range from $13-35 \%$ in SLE patients compared to general population with risk factors being first pregnancy, nulliparity, renal disease, low complement levels, hypertension and dsSDNA positivity. Patients with lupus nephritis have the highest risk with some studies showing rates up to $66 \%$. Pregnancy loss and stillbirth are also higher in SLE, with a rate of up to $20 \%$ with risk factors being SLE activity and antiphospholipid syndrome2. Preterm birth is notably increased in SLE with rates up to $30 \%$ with risk factors being hypertension, SLE activity and higher prednisolone doses2. Finally, low birth weights less than 10th percentile are also found in $10 \%$ of live-births in women with SLE [21].

Whilst the diagnosis of SLE was not obvious during our patients' episode of pre-eclampsia, presence of preeclampsia is a significant marker of her sub-acute presentation of SLE. Although pregnancy is not contraindicated in SLE patients, careful counseling and monitoring of patient through conception, pregnancy and post-partum period is important. Should our patient become pregnant again, remission of SLE activity 6 months prior to conception and careful co-ordination of the high-risk pregnancy with a multidisciplinary team approach is key. The connection between antiphospholipid syndrome and SLE is also important. About 40\% SLE patients have positive antiphospholipid antibodies although development of secondary antiphospholipid syndrome in SLE is slightly less common [22]. Antiphospholipid syndrome is characterized by arterial and venous thrombosis and multiple pregnancy complications including pre-eclampsia, fetal death and placental insufficiency [23].

The specific management of SLE largely depends on the predominant organ system that is involved but a large evidence base has suggested that all patients should be on hydroxychloroquine unless contraindicated [24-26]. A large systematic review revealed that hydroxychloroquine has multiple benefits including reducing flares, protecting in against organ damage, reducing thrombosis and increasing long term survival24. Other studies have also revealed less skin and integument damage with the use of hydroxychloroquine24-26. Lupus nephritis treatment depends usually on the features found at renal biopsy (Table 3) [27,28]. Generally, aggressive immunosuppressive treatment is reserved for classes III and IV with active lesions.
Although the effects of treatment on all-cause mortality was difficult to elicit, KDIGO guidelines for lupus nephritis suggested that induction therapy should consist of corticosteroids and another agent. Whilst cyclophosphamide and MMF both lead to similar rates of disease remission and reduction of time to doubling of creatinine, MMF had lower risk of ovarian failure. Therefore, MMF is the preferred treatment in women of child bearing age, like in our case. For maintenance therapy, evidence shows MMF being superior compared to other agents like azathioprine which increases rates of renal relapse and leucopenia [29].

After appropriate treatment, patients usually go into remission. Rarely, some patients do not require further treatment after the initial presentation but most lead a relapsing and remitting disease course. The prognosis of SLE varies significantly between individuals and depends on various factors with poor prognostic factors being the presence of renal disease, co-existing hypertension, male sex, black race and presence of antiphospholipid syndrome. Although the overall survival has improved in the last decade owing to increased disease recognition and prompt treatment, SLE still has higher mortality rates up to 5 times more than the general population [30].

In summary, the presentation of SLE in the postpartum period is important to note in a young patient with strong family history of autoimmune disease. It is also important to understand that SLE presents heterogeneously with multiple organ involvement with varying severity. Therefore, prompt diagnosis with thorough clinical assessment, laboratory autoimmune panel testing and management of organ disease with an appropriate level of immunosuppression is crucial, to improve the overall prognosis. Finally, it is important to understand the close interaction between pregnancy and SLE with its multiple negative effects on both maternal and fetal morbidity and mortality. The overall management of an individual with SLE should be carried out in a multidisciplinary team involving physicians of different specialties, nursing and allied health staff and psychologists.

\section{References}

1. Iozza I, Cianci S, Natale AD, Garofalo G, Giacobbe AM, et al. (2010) Update on systemic lupus erythematosus pregnancy. J Prenat Med 4(4): 67-73.

2. Clowse M (2007) Lupus activity in Pregnancy. Rheum Dis Clin North Am 33(2): 237. 
3. D Cruz DP, Khamashta MA, Hughes GR (2007) Systemic Lupus Erythromatosus. The Lancet 369(9561): 587-596.

4. Tench CM, McCurdie I, White PD, D'Cruz DP (2000) The prevalence and associations of fatigue in systemic lupus erythematosus. Rheumatology 39(11): 1249-1254.

5. Grossmann JM (2009) Lupus Arthritis. Best Pract Res Clin Rheumatol 23(4): 495-506.

6. Hoover PJ, Costenbader KH (2016) Insights into the Epidemiology and Management of Lupus Nephritis from the U.S Rheumatologist's Perspective. Kidney Int 90(3): 487-492.

7. Yung S, Chan TM (2015) Mechanisms of Kidney Injury in Lupus Nephritis- the Role of Anti-dsDNA Antibodies. Front Immunol 6: 475.

8. Keane M, Lynch JP (2000) Pleuropulmonary manifestations of systemic lupus erythematosus. Thorax 55(2): 159-166.

9. Miner J, Kim AH (2014) Cardiac Manifestations of systemic lupus erythematosus. Rheum Dis Clin North Am 40(1): 51-60.

10. Tian XP, Zhang X (2010) Gastrointestinal involvement in systemic lupus erythematosus: Insight into pathogenesis, diagnosis and treatment. World J Gastroenterol 16(24): 2971-2977.

11. Giannouli S, Voulgarelis M, Ziakas PD, Tzioufas AG (2006) Anaemia in systemic lupus erythematosus: from pathophysiology to clinical assessment. Ann Rheum Dis 65(2): 144-148.

12. Jeffries M, Hamadeh F, Aberle T, Glenn S, Kamen DL, et al. (2008) Haemolytic anaemia in a multi-ethnic cohort of lupus patients: a clinical and serological perspective. Lupus 17(8): 739-743.

13. Michel M, Chanet V, Dechartres A, Morin AS, Piette JC, et al. (2009) The spectrum of Evans syndrome in adults: new insight into the disease based on the analysis of 68 cases. Blood 114(15): 3167-3172.

14. Gomard-Mennesson E, Ruivard M, Koenig M, Woods A, Magy N, et al. (2006) Treatment of isolated severe immune haemolytic anaemia associated with systemic lupus erythematosus: 26 cases. Lupus 15(4): 222-231.
15. Gulinello M, Wen J, Putterman C (2012) Neuropsychiatric symptoms in Lupus. Psychiatr Ann 42(9): 322-328.

16. Jones A, Giles I (2016) Fertility and pregnancy in systemic lupus erythematosus. Ind J Rheumatol 11(6): 128-134.

17. Bermas B, Sammaritano LR (2015) Fertility and pregnancy in rheumatoid arthritis and systemic lupus erythematosus. Fertil Res Pract 1: 13.

18. Meehan R, Dorsey JK (1987) Pregnancy among patients with systemic lupus erythematosus receiving immunosuppressive therapy. J Rheumatol 14(2): 252258.

19. Lima F, Buchanan NMM, Khamashta MA, Kerslake S, Hughes GRV (1995) Obstetric outcome in systemic lupus erythematosus. Semi Arthritis Rheum 25(3): 184-192.

20. Doria A, Cutolo M, Ghirardello A, Zen M, Villalta D, et al. (2012) Effect of pregnancy on serum cytokines in SLE patients. Arthritis Res Ther 14(2): R66.

21. Poon L, Tan MY, Yerlikaya G, Syngelaki A, Nicolaides KH (2016) Birth weights in live births and stillbirths. Ultrasound Obstet Gynaecol 48(5): 602-606.

22. Unlu O, Zuily S, Erkan D (2016) The clinical significance of antiphospholipid antibodies in systemic lupus erythematosus. Eur J Rheumatol 3(2): 75-84.

23. Abrahams V, Chamley L, Salmon J (2017) Antiphospholipid Syndrome and Pregnancy: Pathogenesis to Translation. Arthritis Rheumatol 69(9): 1710-1721.

24. Ruiz-Irastorza G, Ramos-Casals M, Brito-Zeron $\mathrm{P}$, Khamashta MA (2010) Clinical efficacy and side effects of antimalarials in systemic lupus erythematosus: a systematic review. Ann Rheumatol Dis 69(1): 20-28.

25. Akhavan P, Su J, Lou W, Gladman DD, Urowitz MB, et al. (201) The early protective effect of hydroxychloroquine on the risk of cumulative damage in patients with systemic lupus erythematosus. J Rheumatol 40(6): 831-841.

26. Pons-Estel G, Alarcón GS, González LA, Zhang J, Vilá LM, et al. (2010) Possible protective effect of hydroxychloroquine on delaying the occurrence of 
integument damage in lupus: LXXI, data from a multiethnic cohort. Arthritis care Res 62(3): 393-400.

27. Hahn B, McMahon MA, Wilkinson A, Wallace WD, Daikh DI, et al. (2012) American College of Rheumatology Guidelines for Screening, Treatment, and Management of Lupus Nephritis. Arthritis Care Res 64(6): 797-808.

28. Wilhelmus S, Bajema IM, Bertsias GK, Boumpas DT, Gordon C, et al. (2016) Lupus Nephritis management guidelines compared. Nephrol Dial Transplant 31(6): 904-913.

29. KDIGO Glomerulonephritis Gudeline updateEvidence Summary. Lupus Nephritis.

30. Singh R, Yen EY (2018) SLE mortality remains disproportionately high, despite improvements over the last decade. Lupus 27(10): 1577-1581. 